\title{
Automatic detection of language impairment
}

\author{
Hali Lindsay ${ }^{1}$, Johannes Tröger, Nicklas Linz ${ }^{1}$, Jan Alexandersson ${ }^{1}$, \\ Johannes Prudlo ${ }^{2}$ \\ ${ }^{1}$ German Research Center for Artificial Intelligence (DFKI), Germany \\ ${ }^{2}$ Department of Neurology, University of Rostock and DZNE, Germany \\ https://doi.org/10.36505/ExLing-2019/10/0033/000395
}

\begin{abstract}
Amyotrophic Lateral Sclerosis (ALS) is a progressive neurodegenerative disease that presents with cognitive impairment (CI) in up to $50 \%$ of cases. In this study, we consider 17 healthy controls' and 61 ALS patients'-16 with CI and 45 without CIperformances on the cookie theft picture description task. Measures of language impairment are automated and used to evaluate the entire ALS population. Results show that both ALS patients with and without CI show statistically significant differences from healthy controls over four language features.
\end{abstract}

Key words: Amyotrophic Lateral Sclerosis, language impairment

\section{Introduction}

Amyotrophic Lateral Sclerosis (ALS) is a muscle degenerative disease. An estimated $1 / 3$ to $1 / 2$ of cases present with mild to severe cognitive impairment (CI) primarily affecting executive functioning. Current literature suggests that mild language impairment is present-and may even be more prevalent than executive impairment - that cannot be attributed to declining motor function, social withdrawal, or executive dysfunction (Taylor et al., 2013). This paper aims to use automated, reproducible methods for identifying the presence of language impairment in ALS patients with and without CI.

\section{Methods}

Data

German Cookie Theft Picture descriptions from 61 ALS patients (age range $=31-79$ years; 22 female) and 17 age-, gender- and education-matched controls (age range $=44-80$ years; 6 female) were recorded and manually transcribed from audio files with Praat (Boersma \& Weenink, 2019) by trained computational linguists following the CHAT protocol (MacWhinney, 1991). ALS patients were recruited from the university hospitals and DZNE sites in Rostock, Germany and Magdeburg, Germany and gave written consent; the study was conducted according to the Declaration of Helsinki and approved by the local ethics committees. Individuals with ALS were sorted into two groups; 16 with CI ( $A L S_{c i}$, age range $=40-76$ years; 7 female) and 45 without CI $\left(A L S_{\text {nor }}\right.$, age range $=31-79$ years; 15 female). To screen for CI, raw scores of

ExLing 2019: Proceedings of $10^{\text {th }}$ International Conference of Experimental Linguistics, 25-27 September 2019, Lisbon, Portugal 
134 H. Lindsay et al.

the Montreal Cognitive Assessment (MoCA)—a common test used for screening cognitive ability—-were compared to normalized German values for every subject. If a person scored below the fifth percentile they were considered for the $A L S_{\text {ef }}$ group (Strong et al., 2009). No healthy controls (HC) scored below the 5 th percentile for the MoCA.

\section{Features}

For general measures of production, the total number of words (\# $\#_{\text {words }}$ ) and the total number of sentences (\# $\#_{\text {sentences }}$ ) were counted from each sample. To evaluate language function, we considered four measures of performance:

(1) Mean words per sentence (mean sentence length) is calculated as a naive measure of sentence complexity.

(2) Mean dependency length (mean dependency length) is a proxy of syntactic complexity. The average dependency length over all utterances in a person's CTP task is calculated. Dependencies are determined from the parse tree provided by the SpaCy German language models (Honnibal, Johnson 2015). A lower mean dependency compared to healthy controls would indicate difficulty in constructing complex sentences indicating language impairment.

(3) The ratio of open to closed class words (open:closed) is calculated where each word in a production is tagged with a part-of-speech (POS) using German SpaCy language models (Honnibal, Johnson 2015). Closed class words are considered to be conjunctions, demonstratives, prepositions, determiners, and pronouns; all other tags are considered as open class words. A high ratio for open:closed would be in line with agrammatic aphasia (Kim, Thompson, 2004) whereas a low ratio for open:closed may reflect the verb deficits identified by (Bak, Chandran 2011).

(4) Complexity index (Complexity Index) is a proxy of language ability that can be computed from spontaneous speech. Taylor et al. (2013) showed this measure to be useful in identifying language impairment in an ALS population. To calculate CI, responses are broken down into independent and dependent (subordinate or nonfinite) clauses (Hersen 2004). For this paper, the process was automated by using German language models from the Stanford parser (Rafferty, Manning 2008) to produce a parse tree for each response. Independent clauses were considered from the root tag and dependent clauses were collected from the $S$ tag. The following was computed from each participant's CTP task recording:

$$
C I=\frac{s_{\text {count }}+\text { Root }}{\text { Roun }}
$$




\section{Results}

For the statistical analysis, a one-way between-subjects Analysis of Variance was computed for each language feature for the HC, $A L S_{\text {nor }}$ and $A L S_{\text {ei }}$ groups. Significant effects across all groups were found for all language function features; mean sentence length $(\mathrm{F}(2,74)=0.50, \mathrm{p}<0.001)$; mean dependency length $(\mathrm{F}(2,74)=0.38, \mathrm{p}<0.001)$; open:closed $(\mathrm{F}(2,74)=0.29, \mathrm{p}<0.05) ;$ Complexity Index $(\mathrm{F}(2,74)=0.44, \mathrm{p}<0.001)$. No significant effects were found for $\#_{\text {words }}$ and $\#_{\text {sentences: }}$ Between-group posthoc analyses were conducted for all main effects with the Tukey HSD and yielded similar results. There were no significant differences between $A L S_{\text {nor }}$ and $A L S_{\text {ef }}$ but a significant difference between $A L S_{\text {nor }}$ and HC and $A L S_{\text {ei }}$ and HC. Significant results are visualised with box plots in Figure 1.

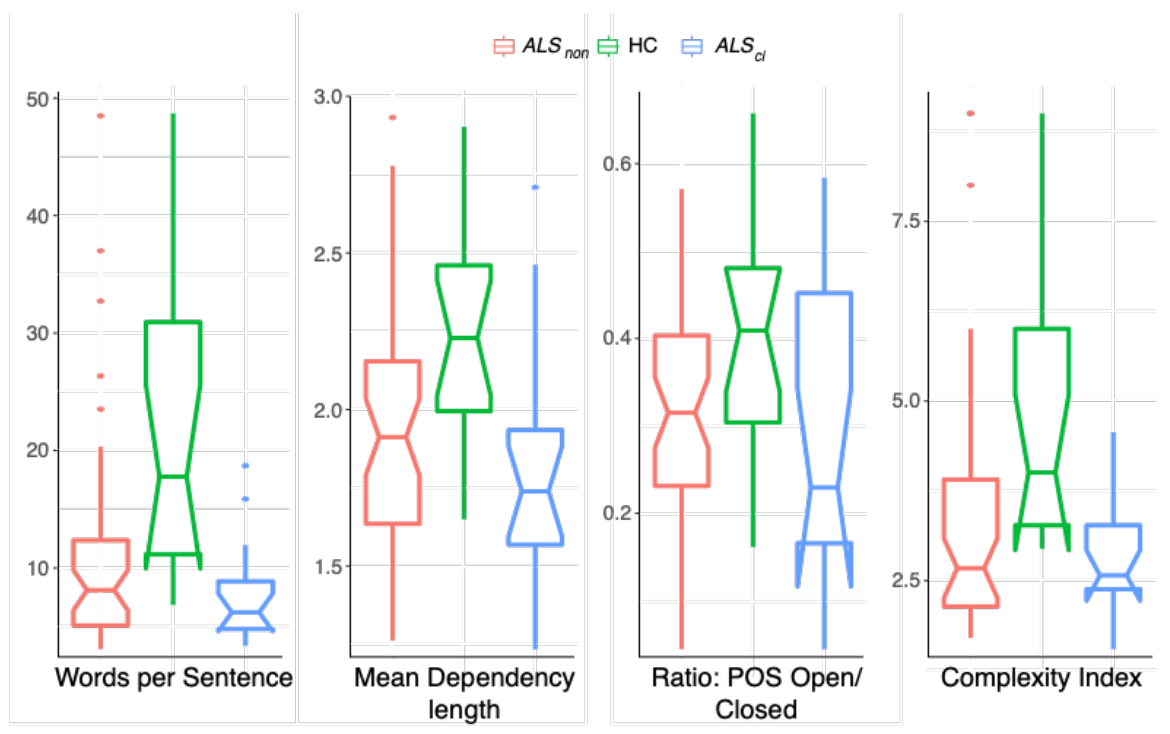

Figure 1. Box plots showing the group performance of each feature

\section{Discussion and conclusion}

Overall, the $A L S_{\text {nor }}$ group produces fewer complex responses than the HC group and performs on-par of those with $A L S_{\text {ef. }}$ There is no significant main difference in $\#_{\text {words }}$ or $\#_{\text {sentences }}$ leading us to believe that this difference is primarily language and not due to a lack of overall production.

Taylor and colleagues (2013) stated that over $40 \%$ of patients impaired on language performed normally on tasks specific to executive impairment and suggested that these groups may even be dissociable. By comparing the $A L S_{e}$ and $A L S_{\text {nor }}$ groups, there is evidence of language impairment in ALS that cannot be attributed to CI. 
136 H. Lindsay et al.

There is evidence, in line with current research-from automated analysis of language features- that both the $A L S_{\text {nor }}$ and $A L S_{\text {e }}$ groups show signs of language impairment. Future work should focus on the prognostic value of language impairment in terms of disease progression.

\section{Acknowledgements}

Thank you to Mario Mina and Marc Sadler for assisting with analysis.

\section{References}

Bak, T., Hodges, J 2004. The effects of motor neurone disease on language: Further evidence. Brain and Language 89, 354-361.

Boersma, Paul, Weenink, David 2019. Praat: doing phonetics by computer [Computer program]. Version 6.1, retrieved 13 July 2019 from http://www.praat.org/.

Hersen, M. 2004. Comprehensive Handbook of Psychological Assessment. John Wiley \& Sons, Inc.

Honnibal, M., Johnson, M. 2015. An Improved Non-monotonic Transition System for Dependency Parsing." Proceedings of the 2015 Conference on Empirical Methods in Natural Language Processing (EMNLP-2015), Lisbon, Portugal. D15-1162 1373 1378. doi: $10.18653 / v 1 / D 15-1162$

Kim, M., Thompson, C.K. 2004. Verb deficits in Alzheimer's disease and agrammatism: implications for lexical organization. Brain and language vol. 88, 1 (2004): 1-20.

MacWhinney, B. 1991. The CHILDES project: Tools for analyzing talk. Lawrence Erlbaum Associates, Inc.

Rafferty, A. Manning, Ch.D. 2008. Parsing Three German Tree. banks: Lexicalized and Unlexicalized Baselines. In ACL Workshop on Parsing German.

Taylor, L., Brown, R., Tsermentseli, S., Al-Chalabi, A., Shaw, C., Ellis, C., Leigh, P., Goldstein, L. 2013. Is language impairment more common than executive dysfunction in Amyotrophic Lateral Sclerosis? Journal of Neurology, Neurosurgery \& Psychiatry, 84, 494-498.

Taylor, L., Brown, R., Tsermentseli, S., Al-Chalabi, A., Shaw, C., Ellis, C., Leigh, P., Goldstein, L. 2013. Is language impairment more common than executive dysfunction in Amyotrophic Lateral Sclerosis? Journal of Neurology, Neurosurgery \& Psychiatry, 84, 494-498.

Tröger, J., Linz, N., König, A., Robert, P., Alexandersson, J. 2018. Telephone-based Dementia Screening I: Automated SVF Assessment. Proceedings of the 12th EAI International Conference on Pervasive Computing Technologies for Healthcare (PervasiveHealth '18). ACM, NYC, NY, USA, 59-66. 